\title{
The roles of HIV-1 specific CD8+ T cell responses and HLA class I alleles on viral control and viral escape in HIV-1 infected Thai individuals
}

\author{
S Buranapraditkun ${ }^{1 *}$, U Hempel $^{2}$, WH Hildebrand ${ }^{3}$, TM Allen $^{2}$, K Ruxrungtham $^{1}$ \\ From AIDS Vaccine 2012 \\ Boston, MA, USA. 9-12 September 2012
}

\section{Background}

Knowledge about the role of specific HLA class I alleles, $\mathrm{CD} 8+\mathrm{T}$ cell responses, and viral escape on viral control have not been well characterized in clade CRF01_AE and Asian ethnics.

\section{Methods}

195 naïve HIV-1 CRF01_AE infected Thai individuals were screened for HIV-1 specific CD8+ T cell responses with a set of 413 OLPs of the HIV-1 proteome by IFNgamma ELISpot assay. Novel epitopes were characterized by HLA restriction and fine epitope mapping. The association of epitopes and/or HLA alleles with low VL level and/ or viral escape was analyzed.

\section{Results}

Thirty-three OLPs were identified as potential novel epitopes. A viral control epitope, RI10 (HIV-protease, previously described in HIV-1 B clade as - B*13 restricted) was found restricted by HLA-A*0203 in Thais. Interestingly, HLA-A*0203+ve patients with RI10 responders had a significantly lower VL than non-responders $(\mathrm{p}=$ 0.0167). This data may support the low VL from loss of viral fitness of RI10. Moreover, the patients exhibiting mutations in RI10 showed no ELISpot responses. Another known HLA-A*1101 epitope, AK11 (in Gagp24) was also identified as a viral control epitope in this study. Of note, there was no significant mutation found in patients expressing $A^{*} 1101$. We also found a novel immunodominant epitope (29\% response rate) restricted by HLA-Cw"0102: YI9 (in Gag-p24) which was associated with viral escape. Mutations at P2 (S278X), P4 (V280X) and P5 (S281G) impaired the Elispot responses, however the P2 anchor S278K mutation had the highest negative impact $(\mathrm{p}=0.0002)$.

\section{Conclusion}

In HIV-1 CRF01_AE infected Thais we characterized three CD8 epitopes (RI10, AK11 and YI9) restricted by HLA-A*0203, - A*1101 and -Cw*0102, respectively. RI10 and AK11, but not YI9, were associated with lower VL and possible control of HIV. Further characterization of those possible novel epitopes is warranted.

\section{Author details}

${ }^{1}$ Chulalongkorn University, Bangkok, Thailand. ${ }^{2}$ Ragon Institute of MGH, MIT and Harvard, Boston, MA, USA. ${ }^{3}$ Department of Microbiology and Immunology, Univ. of Oklahoma, Oklahoma City, OK, USA.

Published: 13 September 2012

doi:10.1186/1742-4690-9-S2-P248

Cite this article as: Buranapraditkun et al:: The roles of HIV-1 specific CD8+ T cell responses and HLA class I alleles on viral control and viral escape in HIV-1 infected Thai individuals. Retrovirology 2012 9(Suppl 2): P248.

${ }^{1}$ Chulalongkorn University, Bangkok, Thailand

Full list of author information is available at the end of the article

(c) 2012 Buranapraditkun et al; licensee BioMed Central Ltd. This is an Open Access article distributed under the terms of the Creative 